\title{
Approche agro-pédologique des zones pastorales sur calcschistes sédimentaires (Beaufortain-Alpes du Nord). I. Caractères de l'altération, propriétés hydriques et porales associées
}

\author{
F van Oort * et JM Dorioz **
}

INRA, station de sciences du sol, route de Saint Cyr, 78000 Versailles, France

(Reçu le 8 juin 1989; accepté le 26 février 1991)

\begin{abstract}
Résumé - En altitude, l'altération des calcschistes sédimentaires (calcshales) conduit à des sols contenant une teneur élevée en limon fin, de composition minéralogique essentiellement micacée. La pédogénèse se caractérise sur ce type de roche, par une argilisation importante due à une microdivision des micas, vers la surface du sol. Ce phénomène entraîne une profonde réorganisation de la phase solide, de l'espace poral et en fin de compte, conditionne les propriétés hydriques des sols.

Dans ce premier texte, un ensemble de méthodes de mesure et d'observation est employé pour décrire l'évolution de la géométrie du système de porosité hérité de la roche, après sa décarbonatation. Cette approche permet de visualiser et de quantifier le volume poral total, la taille, la morphologie et la continuité des pores. La gamme de tailles prise en compte s'étend du nm jusqu'au mm. Grâce aux résultats obtenus, il est possible de comprendre les aspects du fonctionnement hydrique qui conditionnent les relations sol-végétation et qui constituent, par conséquent, un facteur clef pour l'exploitation pastorale.

Les propriétés du matériau d'altération perdent de l'importance quand la pente s'accentue; la circulation de l'eau et la topographie deviennent alors prépondérantes pour expliquer la différenciation des sols et des végétations prairiales. Ces interactions seront traitées dans une deuxième partie.
\end{abstract}

milieu alpin / calcschiste sédimentaire / pédogénèse / altérite / système de porosité / propriété hydrique

Summary - Landscape ecology of alpine pastures in a sedimentary calcschist area (Beaufortain, Northen Alps). I. Characteristics of weathering: consequences on hydric and poral behaviour of soils. The weathering of sedimentary calcschist (calcshales) in the Beaufortain mountain pasture region (fig 1), gives rise to soils containing very high amounts of fine $(<5 \mu \mathrm{m})$ micaceous silt particles (fig 2; tables II, III).

Pedogenesis on this type of parent rock is characterized by a significant argilization (physical breakdown of mica particles) towards the soil surface (table II), causing a total reorganization of solid particle arrangement and corresponding pore geometry (fig 2.2, 2.4, 2.5).

A series of physical methods (table I) is used here in order to describe the origin and evolution of the pore geometry during pedogenesis, starting from the pore system inherited from the parent rock after its decarbonization (fig 2.2 and 2.3). These methods enable the observation and the quantification of the porosity in terms of total pore volume, pore size and pore morphology as well as that aspect concerning continuity of the pores. The pore size ranges from the nm to the $\mathrm{mm}$. Analysis of the pore system and the related soil water behaviour (figs 3, 4, 5; table II) performed in different horizons of a representative soil profile indicates the constraints for local agriculture.

On hill slopes the superficial water circulation and topography are major factors explaining soil and vegetation differentiation. Such soil-vegetation-land use interactions will be discussed in a second paper considering typical geomorphological land units.

Alps / sedimentary calcschist / pedogenesis / alterite / porosity system / soil water properties

\footnotetext{
Adresse actuelle des auteurs :

* CRAAG, unité de science du sol, station agropédoclimatique de la zone Caraïbes, BP 1232, 97184 Pointe-à-Pitre Cedex, Guadeloupe

** INRA, sciences du sol, institut de limnologie, BP 511, 74203 Thonon-les-Bains Cedex, France
} 


\section{INTRODUCTION}

La végétation est au centre de nombreux diagnostics concernant les propriétés agronomiques des milieux de haute altitude. Les déterminants climatiques, lithologiques et pédologiques sont souvent abordés soit à l'échelle de la station, soit au contraire à l'échelle des massifs entiers. Ainsi, de ce dernier point de vue, on distingue dans les Alpes de grands ensembles biogéographiques (Veyret et Veyret, 1979; Ozenda, 1985). Ce découpage en macrorégions, qui recoupe une réalité biologique et humaine complexe, peut se révéler utile pour une zonation agricole. II existe, par exemple, des correspondances entre types de massifs et mode d'utilisation des aipages (Gardelle et Guet, 1968; Dorioz, 1987), entre certaines limites bioclimatiques et propriétés des prairies (Fleury et al, 1985).

Pour les praticiens, ces approches sont insuffisantes. L'essentiel des questions concrètes relatives aux aptitudes du milieu physique (marges de progrès, risque de dégradation, zonages, etc), se pose en fait à des niveaux d'organisation intermédiaires, ceux qui s'étendent de la petite région à l'unité d'exploitation. Pour ces niveaux de diagnostic il faut disposer en particulier, d'un minimum de compréhension des relations solpaysage (Tricart, 1978; Barthes et Bornand, 1986), et des règles de répartition des paramètres agropédologiques. C'est pour acquérir ce fond de connaissance et établir un raisonnement sur les potentialités que nous avons entrepris l'analyse des mécanismes d'organisation des paysages (Naveh et Lieberman, 1984) dans divers territoires pastoraux des Alpes du Nord. Afin d'obtenir des informations utilisables aux diverses échelles pertinentes pour l'aménagement agricole, nous avons couplé l'analyse des relations sol-paysage à des études sur le fonctionnement de sols types.

Une publication précédente traite de la répartition des ressources végétales dans un alpage remarquable par sa diversité lithologique (Dorioz et Party, 1987). Ce travail, associé aux typologies disponibles pour les groupements végétaux et les valeurs pastorales (Gensac, 1979; Richard et Pautou, 1982; Vertes, 1983; Dubost et Bornard, 1986; etc), constitue une base suffisante pour caractériser les herbages. Parallèlement, nous disposons de quelques travaux sur les sols sous prairies de montagne (Cabidoche, 1979; Legros et al, 1980; Legros et al, 1987). Mais les recherches pédologiques entreprises jusqu'alors privilégient les secteurs peu anthropisés et les roches mères "modèles" (granite, calcaire dur, etc). Le cadre de ce travail est bien différent puisqu'il s'agit d'études de sols prairiaux dans un vaste massif schisteux très homogène du point de vue lithologique (les calcschistes sédimentaires ou calcshales).

Pour caractériser le fonctionnement de ces sols, nous employons différentes méthodes de laboratoire destinées à définir certains aspects des propriétés physiques. Sont pris en compte, à la fois l'origine du système de porosité et les modifications de l'organisation du matériau qui se manifestent, lors de la pédogénèse dans les sols en place, du bas vers le haut des profils. Les informations obtenues sont confrontées avec l'étude des propriétés hydriques des différents horizons. On obtient ainsi des éléments importants pour analyser et comprendre les conséquences spécifiques de la pédogénèse sur ce type de roche, en terme de contraintes pour la végétation et les activités pastorales ( $2^{e}$ partie).

\section{CADRE GÉOGRAPHIQUE ET EXPÉRIMENTAL}

\section{Le terrain d'étude : le Beaufortain}

Le Beaufortain (fig 1) se situe à l'extrémité nord-est du massif cristallin de Belledonne. II est soumis à une variante humide du climat montagnard. Le socle cristallin hercynien affleure essentiellement au sud-ouest. Ailleurs, il est recouvert de fragments de la série sédimentaire du Lias. Ces roches sédimentaires engendrent des reliefs relativement peu accidentés, favorables au développement des activités agricoles.

L'élevage laitier à vocation fromagère de qualité, constitue le fondement de l'agriculture du Beaufortain. Bien que les systèmes d'exploitation se soient largement diversifiés depuis 1950, les alpages (ou estives) conservent une place importante dans le fonctionnement de nombreuses exploitations de la région (Huguet et Roybin, 1984). En raison d'un manque de terres dans la vallée, on constate même un mouvement de remise en valeur d'unités pastorales abandonnées.

La surface pastorale d'altitude (>1500 m) représente $50 \%$ de la superficie totale du massif. Les prairies sont situées pour l'essentiel sur des sols développés à partir de matériaux assez homogènes issus de l'altération des calcshales (Van Oort, 1984). Selon Legros et al (1980), ce type de matériau recouvre environ 800000 ha 


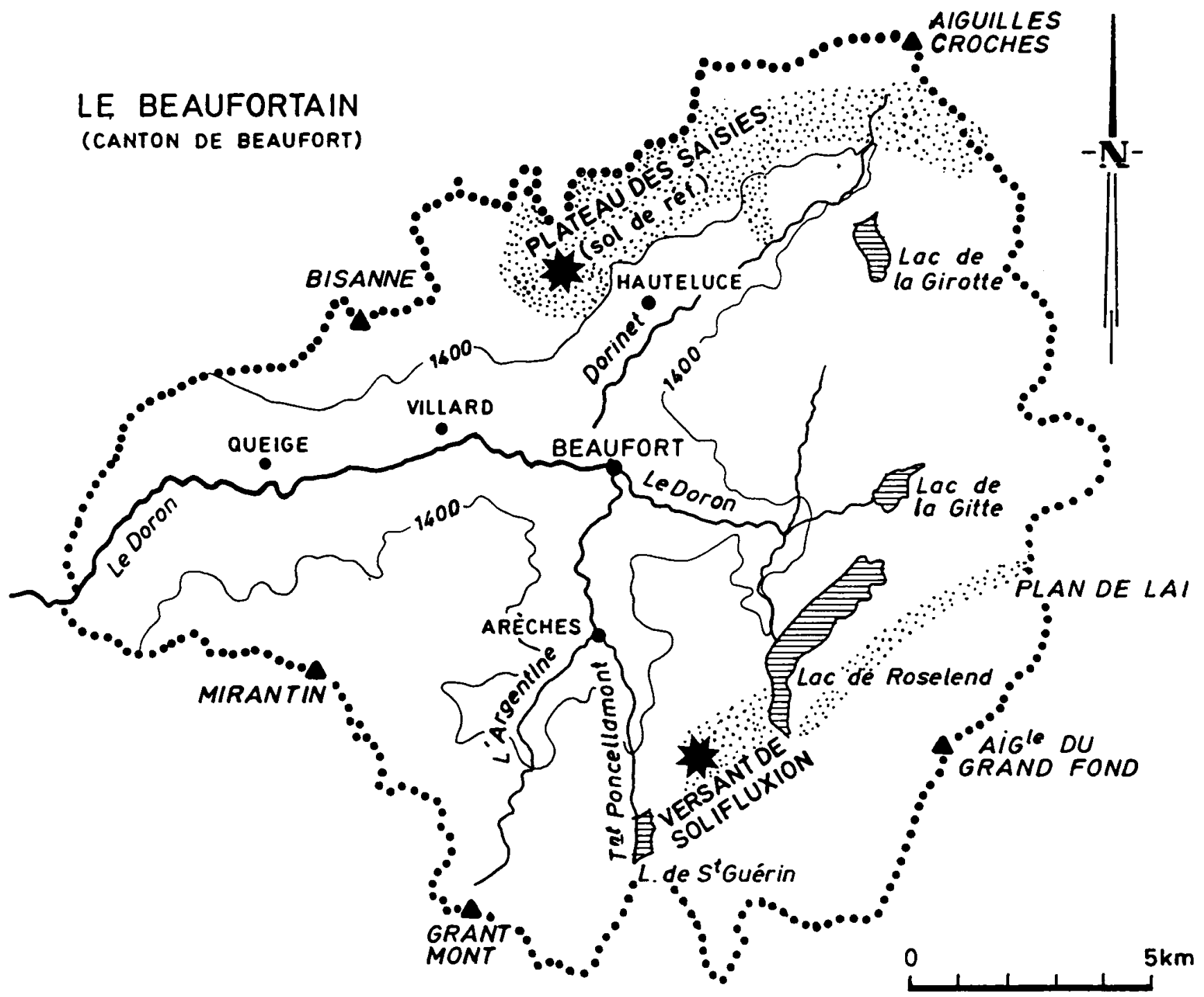

Fig 1. Carte schématique du Beaufortain avec localisation du sol de référence ( $1^{\text {re }}$ partie) et des sites d'étude $\left(2^{\theta}\right.$ partie).

dans les Alpes françaises. Tout ceci explique l'intérêt que nous avons porté à ce type de territoire.

\section{Méthodes de caractérisation des propriétés physiques des sols}

Les propriétés physiques d'un matériau de sol sont déterminées non seulement par la nature minéralogique des constituants, mais aussi par leur arrangement et par l'espace poral qui en résulte. Ces propriétés dépendent donc en grande partie des relations entre les différents niveaux d'organisation du sol, depuis le feuillet de phyllosilicate jusqu'à l'agrégat. Pour caractériser des paramètres physiques on doit donc utiliser un en- semble cohérent de méthodes de mesure et d'observation (tableau I).

Compte tenu des modifications qui se manifestent dans l'organisation de la phase solide au cours de la dessiccation (Tessier, 1984; Bruand, 1985) une telle caractérisation nécessite des procédés de déshydratation adaptés. Enfin, nous retiendrons pour classer les divers types de pores, la classification de van Oort (1984).

\section{Arrangement des particules élémentaires}

Cet arrangement et l'organisation des structures aux échelles les plus fines sont observés en microscopie électronique à balayage (MEB). Les échantillons étudiés sont non remaniés, d'abord 
Tableau I. Domaine d'application et détails techniques des différentes méthodes d'étude de l'organisation structurale des sols (d'après Van Oort et al, 1987, modifié).

\begin{tabular}{|c|c|c|}
\hline Méthode & Type et taille d'échantillon & Procédés de préparation \\
\hline $\begin{array}{l}\text { Microscope électronique } \\
\text { à transmission }\end{array}$ & lames ultraminces $\left(\mu \mathrm{m}^{2}\right)$ & $\begin{array}{l}\text { échange eau/acétone, point critique } \\
\text { inclusion résine de Spurr }\end{array}$ \\
\hline $\begin{array}{l}\text { Microscope électronique } \\
\text { à balayage }\end{array}$ & agrégats $\left(\mathrm{mm}^{3}\right)$ & cryodessiccation, lyophylisation \\
\hline Loupe binoculaire & échantillons naturels $(\mathrm{cm})$ & - \\
\hline $\begin{array}{l}\text { Microscope polarisant } \\
\text { Micromorphométrie }\end{array}$ & lames minces $(6 \times 8 \mathrm{~cm})$ & $\begin{array}{l}\text { échange eau/acétone, imprégnation } \\
\text { résine }\end{array}$ \\
\hline $\begin{array}{l}\text { Diffractométrie des } \\
\text { rayons } X\end{array}$ & $\begin{array}{l}\text { fraction }<2 \mu \mathrm{m}, \text { pâte humide } \\
\text { orientée ou poudre sèche }\end{array}$ & $\begin{array}{l}\text { extraction argile, saturation avec un cation } \\
\left(\mathrm{Mg}^{2+}, \mathrm{K}^{+}\right) \text {; intercalation avec } \\
\text { des produits organiques; chauffage }\end{array}$ \\
\hline $\begin{array}{l}\text { Porosimétrie à } \\
\text { mercure }\end{array}$ & agrégats $\left(1-5 \mathrm{~mm}^{3}\right)$ & $\begin{array}{l}\text { cryodessiccation/lyophilisation } \\
\text { (ou point critique) }\end{array}$ \\
\hline $\begin{array}{l}\text { Détermination indice } \\
\text { d'eau/indice des vides }\end{array}$ & «mottes» non remaniées $\left(\mathrm{cm}^{3}\right)$ & - \\
\hline Conductivité hydraulique & cylindres $\left(250 \mathrm{~cm}^{3}\right)$ & prélèvement d'échantillons non perturbés \\
\hline
\end{tabular}

amenés à une valeur donnée de potentiel de l'eau, puis déshydratés par la méthode de la congélation-lyophilisation (Tessier et Berrier, 1979). L'emploi de cette méthode de déshydratation sur ce type de matériau semble autorisé, car nous n'avons pas observé les microfissures signalées par certains auteurs comme étant des artefacts dus à la congélation (Bruand, 1985; Bruand et Tessier, 1987). L'organisation de structures plus macroscopiques est observée sur des lames minces de grande taille $(8 \times 6$ $\mathrm{cm}$ ), réalisées à partir d'échantillons imprégnés et préparés selon la méthode de Miedema et al (1974).

\section{L'étude de l'espace poral}

Elle est basée sur une série de méthodes complémentaires. La valeur de la porosité totale, $\left(P_{t}\right)$, est calculée à partir de la masse volumique du solide ( $s$ ), déterminée au pycnomètre à eau et de la masse volumique du sol (d), déterminée sur motte à un potentiel donné de l'eau (Monnier et al, 1973) tel que $P_{t}=[(s-d) / s] \times 100 \%$.

La porosimétrie à mercure (Vachier et al, 1979) utilise la relation entre le rayon de cons- triction des pores (notion de rayon équivalent, $R_{e ́ q}$ ) et la pression $P$ qu'il faut exercer sur le mercure pour qu'il y pénètre. Cette relation s'écrit $P=2\left(\gamma_{\mapsto g}\right)(\cos \Delta \alpha) /\left(R_{\dot{\alpha} q}\right)$ avec $\gamma_{H g}$ tension superficielle de mercure (soit $0,48 \mathrm{~N} . \mathrm{m}^{-1}$ ), $\alpha$ angle de contact entre le mercure et la phase solide (soit $141^{\circ}$ ). La méthode donne une courbe de distribution des volumes poraux en fonction de $R_{\dot{e} q}$. L'analyse d'image (Ismaïl, 1975; Chrétien, 1987) complète l'étude du système poral en permettant de quantifier la surface porale totale et la distribution des macropores sur lames minces.

\section{Les propriétés de rétention en eau d'un sol}

Elles sont plus particulièrement liées aux forces de capillarité et aux forces d'adsorption dues aux particules de solide, le potentiel matriciel $(\mathrm{m})$.

Les variations de la répartition de l'eau et de l'air dans un échantillon ainsi que l'évolution de son volume (volume apparent en $\mathrm{cm}^{3} \cdot \mathrm{g}^{-1}$ ), peuvent être déduites des courbes teneur en eau/pF et volume apparent/pF $(p F=\log (m)=\log (p)$, avec $p$ : pression en mbar). Ici encore la loi de Jurin permet de relier pression et taille des pores. $p=0,15 / R_{\dot{e q}}$ ( $p$ en mbar et $R_{e q}$ en $\mathrm{cm}$ ). 
Dans ce travail les quantités d'eau, de vides et d'air sont exprimées en $\mathrm{cm}^{3}$ par $\mathrm{cm}^{3}$ de solide et appelées respectivement: indice d'eau $\left(e_{w}\right)$, indice des vides $(e)$ et indice d'air $\left(e_{a}\right)$.

\section{Les mesures de conductivité hydraulique en milieu saturé (Hillel, 1971)}

Elles sont réalisées au laboratoire sur cylindres (diamètre $8 \mathrm{~cm}$, volume $250 \mathrm{~cm}^{3}$ ) en tenant compte de l'anisotropie du système poral dans un milieu schisteux (Dejou et al, 1971; Cabidoche, 1979), ces mesures complètent ce dispositif analytique et doivent permettre de mieux comprendre le fonctionnement du sol dans le milieu naturel.

\section{CARACTÉRISTIQUES PHYSICO-CHIMIQUES DU MATÉRIAU D'ALTÉRATION DES CALCSCHISTES}

\section{Le profil pédologique type}

Le sol de référence s'est développé en place en position (sub)horizontale (situation de plateau), sous prairie d'alpage soumise à une pâture extensive (Nardetum). Il présente les caractéristiques spécifiques de la pédogénèse sur calcshale. II montre une succession d'horizons $A$, (B), (B)C, et $C$ (fig 2.1), qui reposent sur la roche mère calcaire litée $(R)$. La pédogénèse conduisant à ce type de sols a été discutée par Legros et al (1980), le profil décrit en détail par van Oort (1984) et les premiers stades de l'altération, par van Oort et Robert (1988). Nous insisterons ici uniquement sur les évolutions pédologiques affectant les différents horizons du sol. En raison de l'importance des phénomènes intervenant dans la courte transition entre les horizons $C$ et (B)C, certains types d'analyse (granulométrie détaillée, porosimétrie à mercure) ont porté sur $2 \mathrm{ni}$ veaux intermédiaires, dénommés $(B) C$ " et $(B) C$ '.

\section{Origine du matériau d'altération}

L'abondance des précipitations et les températures moyennes assez basses favorisent une décarbonatation intense et rapide de la roche mère. Dans l'horizon d'altération C, l'organisation générale de la roche est conservée mais la cohésion est très faible : il s'agit d'une isaltérite (fig 2.2, 2.3). Les discontinuités macroscopiques corres- pondent aux plans de schistosité de la roche et à l'emplacement de veines de calcite. Leur taille est d'environ $20-50 \mu \mathrm{m}$. Le matériau compris entre ces discontinuités est caractérisé par une porosité très élevée, due à la dissolution de cristaux de calcite. La taille des vides est, soit de l'ordre de quelques $\mu \mathrm{m}$ (grains de calcite micritiques), soit de quelques dizaines de $\mu \mathrm{m}$ (agrégoclastes microsparitiques). Cette altérite subit ensuite plusieurs transformations, souvent concomitantes. Nous allons les examiner.

\section{Évolutions pédologiques}

L'organisation subhorizontale et assez régulière de l'isaltérite est rapidement modifiée par la pénétration des racines, la circulation de la faune et les agents climatiques. Ainsi, dans l'horizon (B)C des sols, $2-3 \mathrm{~cm}$ seulement au-dessus de l'horizon $\mathrm{C}$, la disposition initiale disparaît (fig 2.4). Dans l'horizon (B) on n'observe ni fissure, ni orientation préférentielle (fig 2.5) : la structure est de type continu avec une tendance microagrégée qui s'exprime très nettement après une dessiccation.

D'un point de vue physique, l'originalité de ce matériau réside dans la finesse et l'homogénéité granulométrique et minéralogique des particules qui le constituent (tableau II). La décarbonatation libère des quantités considérables de particules limoneuses (jusqu'à $85 \%$ ), essentiellement de la taille limon fin. Celles-ci subissent ensuite une microdivision (Legros, 1982), ce qui explique l'augmentation du taux d'argile vers la surface des sols. L'accroissement léger de la teneur en sable dans les horizons de surface s'explique par la présence de petits nodules ferro-manganiques.

Observée en microscopie à balayage (MEB), la fraction argileuse apparaît constituée essentiellement de particules pyllosilicatées planes (fig 2). Les études minéralogiques (van Oort, 1984) confirment que cette fraction est composée de micromicas, de quelques chlorites héritées, et de cristaux de quartz. Compte tenue de la nature peu altérable de ces minéraux, on n'observe pas d'évolution minéralogique notable. Au total, il s'agit d'un matériau peu réactif, avec des faibles valeurs de la capacité d'échange cationique (CEC entre 7 et $10 \mathrm{meq} / 100 \mathrm{~g}$ dans les horizons $B, B(C)$ et $C$; tableau III). Ce matériau se caractérise en outre, pour des raisons minéralogiques (Tessier, 1984), par une faible aptitude à se structurer en agrégats. 


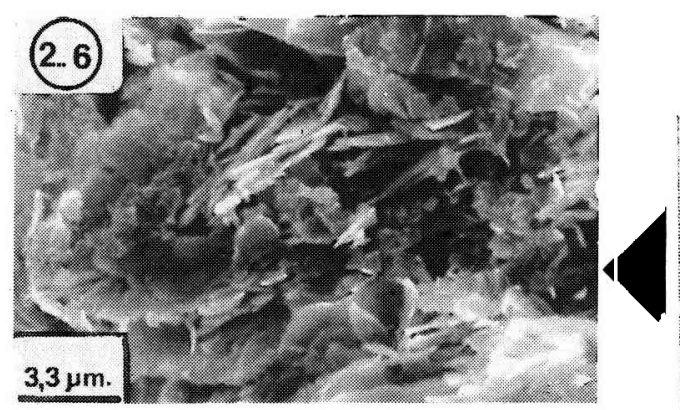

\section{5}
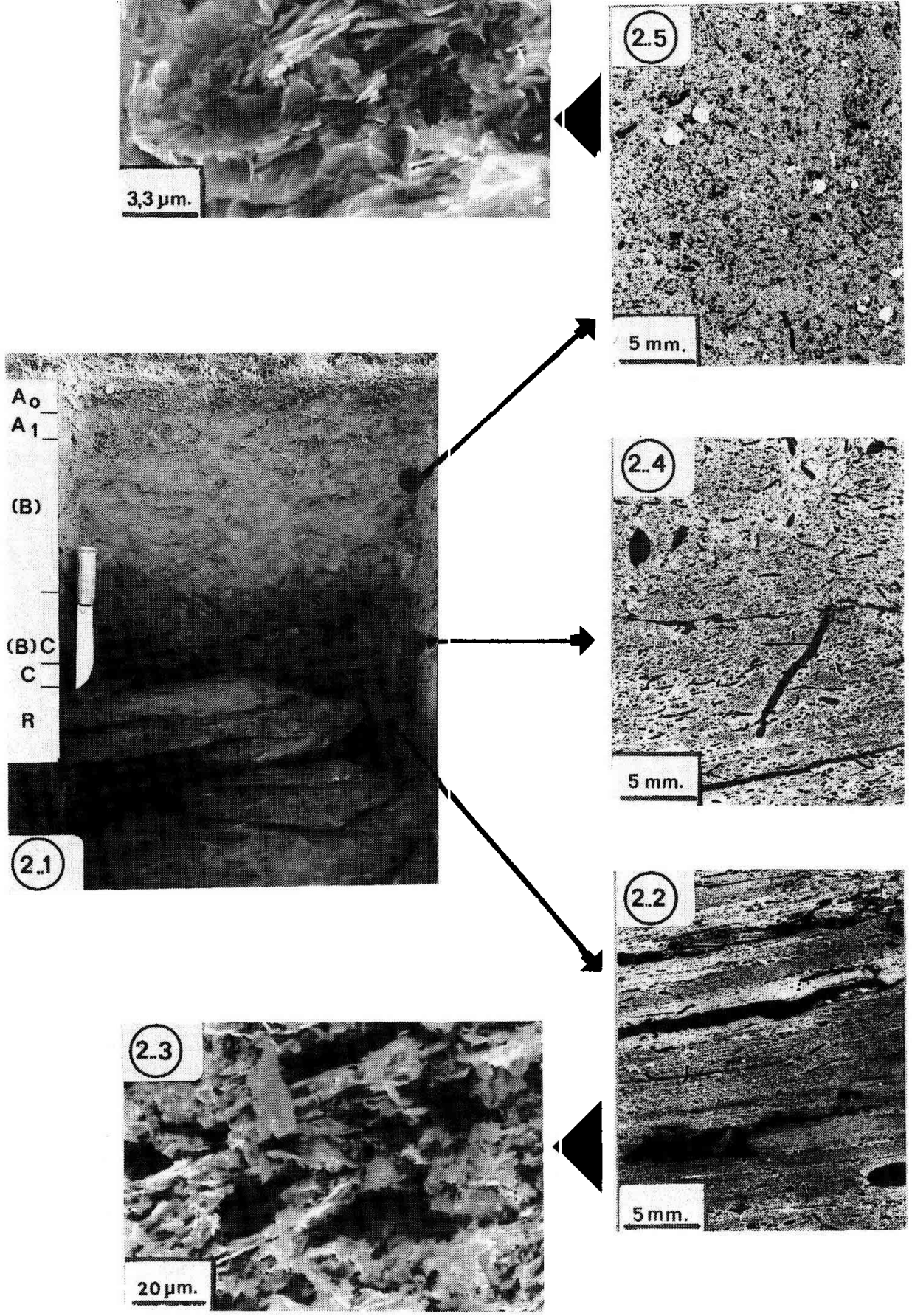

Fig 2. Organisation générale des constituants minéraux du sol de référence développé sur calcshale. $2-1$ : profil pédologique; $2-2$ horizon C (isaltérite : lame mince, Gx2); 2-3: détail de l'horizon C (MEB, Gx480) 2-4; horizon (B)C (Lm, Gx2); 2-5: horizon (B) (Lm, Gx2); 2-6 : détail de l'horizon (B) (MEB, Gx3000); sur les lames minces $(L m)$ les vides sont noirs.

Sur le plan chimique, l'altération des calcshales conduit à des sols désaturés, parfois acides comme dans le cas du sol type (tableau III). Lors du processus d'acidification, $\mathrm{H}^{+}$et surtout $\mathrm{A} \mid{ }^{3}+$ remplacent, sur le complexe d'échange, les éléments basiques lixiviés. le taux de $\mathrm{Ca}^{2+}$ augmente dans l'horizon de surface en raison des remontées biologiques intenses sous prairie. En profondeur, laugmentation de ce même taux traduit la proximité de roches contenant du $\mathrm{CaCO}_{3}$. Dans la différenciation des horizons pédologiques, ces facteurs chimiques ont cependant un rôle mineur, par rapport aux facteurs physiques. 
Tableau II. Analyses physiques des horizons d'un sol développé en place : modifications dans la composition granulométrique, évolution des valeurs de la densité réelle (s) et apparente (d), de la porosité totale ( $P t)$ et de l'indice des vides (e); les valeurs des 3 derniers paramètres sont calculées à pF 2.

\begin{tabular}{|c|c|c|c|c|c|c|c|c|c|c|c|c|}
\hline \multirow[b]{2}{*}{ Horizon } & \multirow[b]{2}{*}{$0 / 2$} & \multirow[b]{2}{*}{$2 / 5$} & \multirow[b]{2}{*}{$5 / 10$} & \multicolumn{3}{|c|}{ Granulométrie $(\mu \mathrm{m})$} & \multirow[b]{2}{*}{$50 / 200$} & \multirow[b]{2}{*}{$200 / 2000$} & \multicolumn{2}{|c|}{ Densité } & \multicolumn{2}{|c|}{ Porosité } \\
\hline & & & & $10 / 20$ & $20 / 30$ & $30 / 50$ & & & $d$ & $s$ & $P t(\%)$ & $e$ \\
\hline A & 36,1 & 18,6 & 15,1 & 10,8 & 3,1 & 0,9 & 5,3 & 10,1 & 1,00 & 2,73 & 63 & 1,73 \\
\hline (B) & 39,1 & 18,6 & 15,2 & 11,2 & 3,2 & 0,7 & 6,0 & 5,2 & 1,02 & 2,77 & 63 & 1,68 \\
\hline$(B) C$ & 21,9 & 18,9 & 23,3 & 23,9 & 6,5 & 1,0 & 2,0 & 2,5 & 1,04 & 2,86 & 63 & 1,75 \\
\hline (B) $\mathrm{C}^{\prime}$ & 17,1 & 18,6 & 24,1 & 26,4 & 7,6 & 2,0 & 2,0 & 2,2 & - & - & - & - \\
\hline (B) C" & 14,5 & 16,1 & 22,1 & 28,8 & 9,7 & 2,1 & 2,5 & 4,2 & - & - & - & - \\
\hline C & 12,0 & 13,9 & 20,8 & 33,9 & 12,9 & 3,7 & 1,5 & 1,3 & 0,63 & 2,85 & 78 & 3,52 \\
\hline
\end{tabular}

Tableau III. Évolution de quelques paramètres physico-chimiques du sol étudié.

\begin{tabular}{|c|c|c|c|c|c|c|c|c|c|}
\hline \multirow[t]{2}{*}{$\begin{array}{l}\text { Profondeur } \\
\qquad(\mathrm{cm})\end{array}$} & \multicolumn{2}{|c|}{$\begin{array}{l}\text { Matière } \\
\text { organique }\end{array}$} & \multirow[t]{2}{*}{$\begin{array}{l}p H \\
\text { eau }\end{array}$} & \multicolumn{5}{|c|}{$\begin{array}{l}\text { Analyse chimique du complexe } \\
\text { d'échange (méq par } 100 \mathrm{~g} \text { de sol) }\end{array}$} & \multirow{2}{*}{$\begin{array}{c}\text { Taux de } \\
\text { saturation } \\
\text { S/T\% }\end{array}$} \\
\hline & $\% M O$ & $C / N$ & & $C E C$ & $\mathrm{Ca}$ & $M g$ & $K$ & $\mathrm{Na}$ & \\
\hline $0-10 \mathrm{~A}$ & 3,6 & 11,4 & 4,6 & 17,3 & 0,9 & 0,25 & 0,15 & 0,07 & $<10$ \\
\hline $10-40\left(B_{1}\right)$ & 1,5 & 4,0 & 4,8 & 9,9 & 0,4 & 0,07 & 0,6 & 0,2 & $<10$ \\
\hline $40-50\left(B_{2}\right)$ & 0,7 & - & 5,0 & 7,3 & 0,4 & 0,05 & 0,04 & 0,02 & $<10$ \\
\hline $50-60(B) C$ & 0,4 & - & 6,6 & 10,7 & 6,2 & 0,10 & 0,02 & 0,01 & 60 \\
\hline
\end{tabular}

\section{CARACTÉRISATION DES PROPRIÉTÉS HYDRIQUES}

Les propriétés hydriques et porales de ce matériau d'altération original ont fait l'objet d'études approfondies. Nous présentons ici les résultats principaux des analyses réalisées sur les différents horizons et leurs conséquences et termes de rétention et de circulation de l'eau.

\section{Microporosité et disponibilité de l'eau}

Les figures 3 et 4 mettent en évidence le comportement hydrique particulier de l'horizon $\mathrm{C}$ par rapport aux horizons $(B) C$ et $(B)$.

\section{Dans I'horizon C}

La forte pente d'eau entre pF 2 et pF 3 (fig 3) correspond, selon la loi de Jurin, à une vidange de pores dont le rayon équivalent est compris entre 15 et $1,5 \mu \mathrm{m}$. Ces valeurs coïncident avec le principal mode de distribution de la taille des pores $(R=7 \mu \mathrm{m})$ mesuré par la porosimétrie à mercure dans cet horizon (fig 4). II s'agit là essentiellement d'une porosité résultant de la dissolution des cristaux de calcite isolés dans la matrice phylliteuse.

\section{Dans les horizons (B)C et (B)}

Pour des contraintes inférieures à pF 3 , on note une faible diminution du volume d'eau et du vo-

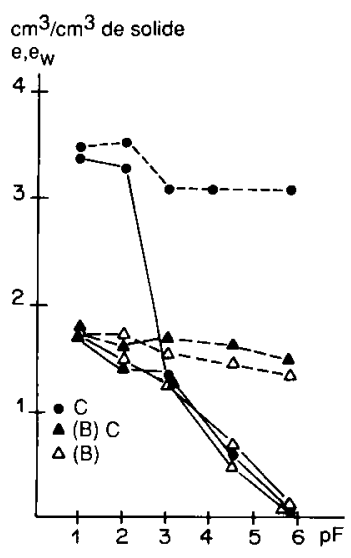

Fig 3. Présentation simultanée de l'évolution des valeurs d'indice d'eau $\left(e_{w},-\right)$ et $(e,---$, mesurées sur mottes non remaniées au cours de la dessication). 


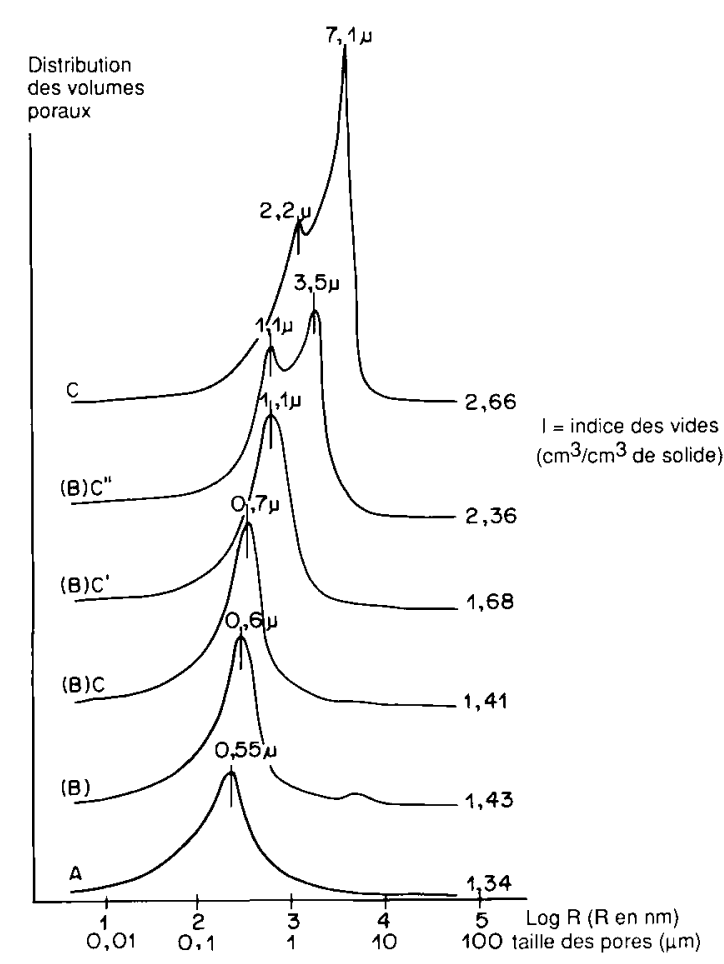

Fig 4. Évolution de la distribution de la taille des pores de bas en haut du profil; mesures par la méthode de porosimétrie à mercure. $(I=$ indice des vides mesurés par cette méthode).

lume poral (fig 3). Ceci traduit l'absence de la classe de pores ayant un rayon équivalent $>1,5$ $\mu \mathrm{m}$ (fig 4). En fait, de $\mathrm{C}$ à (B), on constate la disparition des macro- et mésopores résultant de la décarbonatation, le spectre de porosité étant totalement modifié (fig 4).

À ce premier niveau d'étude, le matériau issu de l'altération des calcschistes se caractérise donc par un système poral à tendance monodimensionnelle, dont la dimension dominante varie selon l'horizon en relation avec la granulométrie et la pédogénèse. On retrouve là des caractéristiques signalées par Chrétien et Pedro (1987) pour les matériaux à fraction granulométrique dominante.

Dans l'isaltérite, on note une forte capacité de rétention en eau et une porosité élevée (tableau II), formée de vides de grande taille; l'eau est facilement disponible pour la végétation, la taille des pores devant permettre sa circulation rapide.

Dans l'horizon (B), la teneur en eau et le volume poral (indice des vides) ont diminué d'environ $50 \%$ par rapport aux valeurs notées dans l'horizon C; le système poral est constitué essentiellement de micropores (rayon équivalent 0,6 $\mu \mathrm{m})$; l'eau retenue dans ces pores est plus difficilement accessible pour les plantes.

\section{Macroporosité et circulation de l'eau}

L'observation microscopique de lames minces (figs 2 et 5) et l'analyse d'image permettent d'évaluer la macroporosité en termes de surface totale et de distribution de la taille et de la forme des pores. Ces caractéristiques sont ensuite confrontées aux mesures de la conductivité hydraulique.

\section{Dans I'horizon C}

II s'agit de vides planaires avec des parois essentiellement droites (figs 2.2 et $5 \mathrm{a}$ ). La distribution des macropores est bimodale avec des modes de diamètres dans les classes 100-300 $\mu \mathrm{m}$ et 500-1 $200 \mu \mathrm{m}$. La classe $1200-3000 \mu \mathrm{m}$ est importante.

La valeur de la conductivité hydraulique est élevée, surtout pour un écoulement latéral (écoulement vertical $\left(K_{\text {sat }}=1,1 \mathrm{~cm} . \mathrm{h}^{-1}\right.$; écoulement latéral $\left.K_{\text {sat }}=36 \mathrm{~cm} \cdot \mathrm{h}^{-1}\right)$. Ceci révèle la continuité du système de macropores et la facilité d'écoulement dans cet horizon situé à la base du profil.
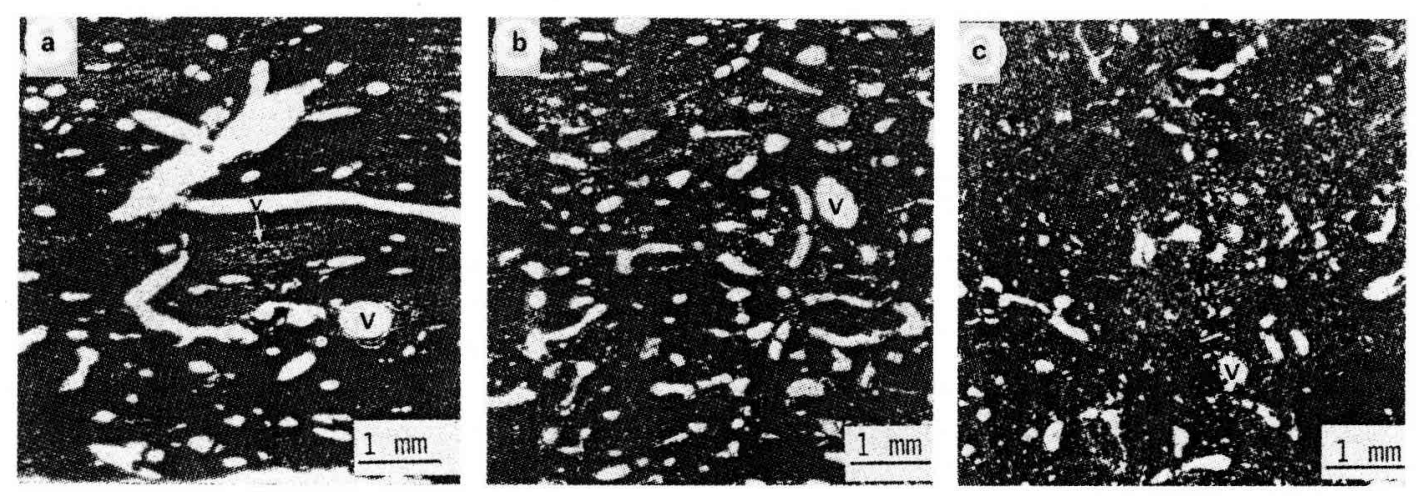

Fig 5. Taille et forme des pores d'origine racinaire dans les différents horizons (lames minces, microscope polarisant, LN, G $\times 10$ ). 5a : horizon $C ; 5 b$ : horizon $(B) C ; 5 c$ : horizon $(B) ;(V=v i d e)$. 


\section{Dans l'horizon (B)}

Le spectre de la macroporosité est totalement différent : les pores sont de type chenal avec des cavités régulières et irrégulières (figs 2.5 et $5 \mathrm{c}$ ). La classe $1200-3000 \mu \mathrm{m}$ a pratiquement disparu et la classe 100-300 $\mu \mathrm{m}$ qui constitue le mode, diminue nettement par rapport à son niveau dans I'horizon C. Enfin, les valeurs de la surface porale totale mesurées sur les lames minces (fig $5 \mathrm{a}$ et $5 b$ ) dans les horizons $C$ et $(B) C$ (respectivement 13,2 et $13,7 \%$ ) s'opposent à celles mesurées en $(B): 6,5 \%$.

La conductivité hydraulique reflète ces changements, avec des valeurs très basses de $K_{\text {sat }}$ dans l'horizon (B), quelle que soit la direction de l'écoulement (écoulement vertical $K_{\text {sat }}=0,04$ $\mathrm{cm} \cdot \mathrm{h}^{-1}$; écoulement horizontal $K_{\text {sat }}=0,08 \mathrm{~cm} \cdot \mathrm{h}^{-1}$ ). L'horizon (B) s'avère très peu perméable, ce qui s'explique par la disparition des macropores hérités de la roche.

\section{Conséquences : Eléments clefs du fonctionnement hydrique}

Dans ces sols développés en place, l'évolution pédologique principale est d'ordre structural. Elle correspond essentiellement à la déstabilisation et la disparition de l'organisation héritée de la roche. La décarbonatation suivie d'une microdivision des particules phyllosilicatées est à l'origine de ce véritable effondrement de l'organisation initiale. C'est l'action des forces d'hydratation qui semble être à l'origine de la microdivision. L'eau en occupant les micropores provoque la désagrégation des phyllosilicates comprimés lors du processus de diagénèse des matériaux sédimentaires (Dejou et al, 1977).

Les modifications structurales se traduisent par une forte baisse de la porosité totale et de la taille moyenne des macro- et des mésopores. Notons à ce propos que l'indice des vides rend mieux compte de la diminution du volume poral que la valeur de la porosité totale (tableau II); entre les horizons $C$ et $(B), P_{t}$ varie de 78 à $63 \%(-20 \%)$, alors que e diminue de 3,25 à 1,73 $\mathrm{cm}^{3} / \mathrm{cm}^{-3}$ de solide $(-51 \%)$. Par ailleurs, la quantité d'eau disponible pour la végétation diminue également très fortement. Ainsi, au-dessus de l'horizon $C$, horizon doté d'une grande réserve en eau, se superpose un horizon (B), quasi imperméable, microporeux et ne libérant son eau qu'à de fortes succions.
L'horizon A conserve l'organisation microscopique de (B) (fig 4), mais avec un drainage meilleur grâce à une structure grumeleuse typique, liée à l'activité des racines.

La mise en évidence du caractère peu perméable de l'horizon (B) par des mesures au laboratoire, fournit des éléments de compréhension du fonctionnement hydrodynamique de ces sols d'alpage. En effet, la circulation de l'eau observée en été est double : superficielle, sous le mat racinaire et profonde, latérale, dans l'isaltérite au contact de la roche dure. À la fonte des neiges, le sol est uniformément saturé mais l'horizon (B) maintient un toucher "sec", par rapport aux couches superficielles et profondes. Pendant l'été, les caractéristiques porales (et chimiques) de cet horizon (B) limitent les possibilités d'absorption de l'eau et d'éléments minéraux par les racines, les réserves principales se trouvant en profondeur, dans l'horizon $\mathrm{C}$.

\section{CONCLUSION}

L'originalité de la pédogénèse sur ces calcschistes réside dans la profonde évolution structurale du matériau et dans son argilisation importante par microdivision. Ceci se manifeste dès la décarbonatation de la roche mère.

Une étude détaillée des modifications de la géométrie du système poral et de la distribution de la taille des particules a permis de fournir des explications sur l'origine et les conséquences de ces transformations.

Les changements dans l'arrangement de la phase solide et de l'espace poral, ont pu être visualisés et quantifiés grâce à l'utilisation d'un ensemble cohérent de méthodes d'observation et de mesures. Compte tenu de leur complémentarité, ces méthodes ont permis de caractériser des pores allant du $\mathrm{nm}$ jusqu'au $\mathrm{mm}$.

Ainsi, entre l'horizon d'altération $C$ et l'horizon (B), on observe l'apparition d'une classe de micropores et une très forte diminution de la porosité totale qui est la conséquence de la quasi disparition des macro- et mésopores de dissolution. La confrontation de ces résultats avec une étude des propriétés hydriques montre, qu'à l'horizon C, caractérisé par une forte capacité de rétention en eau, une grande disponibilité de l'eau et une forte perméabilité, se superpose un horizon (B) à caractère peu perméable et à faible disponibilité de l'eau. 
Les propriétés hydriques et physiques spécifiques du matériau d'altération des calcschistes et les tendances de la pédogénèse d'altitude vont largement influencer la dynamique végétale. Mais cette influence s'exprime par des contraintes agronomiques différentes selon la topographie. La connaissance du matériau permet alors d'expliquer les lois de répartition des propriétés agronomiques dans le milieu. Cette réflexion fera l'objet d'un article suivant.

\section{REMERCIEMENTS}

Cet article a bénéficié des critiques et des conseils de JP Legros (INRA, Montpellier), A Bruand (INRA, Orléans) et M Robert (INRA, Versailles). Le travail présenté a été réalisé dans le cadre du programme de recherche développement sur l'agriculture de montagne (GIS «Alpes du Nord»).

\section{RÉFÉRENCES}

Barthes JP, Bornand M (1986) Cartographie des sols en moyenne montagne calcaire sèche. Séminaires d'agro-météorologie. Toulouse. Colloque INRA $n^{\circ}$ 39, INRA

Bruand A (1985) Contribution à l'étude de la dynamique de l'organisation de matériaux gonflants. Application à un matériau provenant d'un sol argilolimoneux de l'Auxerrois. Thèse Doct spécialité, Univ Paris VII, France, $225 \mathrm{p}$

Bruand A, Tessier D (1987) Étude de l'organisation d'un matériau argileux en microscopie, modification intervenant lors de la déshydratation. In: Micromorphologie des sols (N Fedoroff, LM Bresson, MA Courty, eds), Actes VIle Réunion Int Micromorphologie Sols, Paris, 31-36

Cabidoche YM (1979) Contribution à l'étude des sols de haute montagne. Analyse des relations solsmilieu dans les Pyrénées Occidentales cristallines et calcaires. Thèse Doct spécialité, Univ Montpellier, $158 p$

Chrétien J (1987) Analyse et quantification de l'espace poral de trois sols développés sur granit et mica-schistes : comparaison méthodologique. In: Micromorphologie des sols (N Fedoroff, LM Bresson, MA Courty, eds). Actes VIle Réunion Int Micromorphologie Sols, Paris, 529-536

Chrétien J, Pedro G (1987) Granulométrie, porosité, spectre poral de sols développés sur formation détritiques. Cas des terrasses alluviales de la Saône. Cah ORSTOM, sér Pédol, vol XXIII, 1, 43-54

Dejou J, Guyot J, Pedro G, Chaumont C (1971) Étude sur le rôle du pendage dans l'évolution superficielle des roches schisteuses. Cas de l'altération des micaschistes du Massif central français. Pédologie. Gand, t. XXI, $n^{\circ}$ 3, 343-359
Dejou J, Guyot J, Robert M (1977) Évolution superficielle des roches cristallines et cristallophyliennes dans les régions tempérées, INRA, Paris, $464 \mathrm{p}$

Dorioz JM (1987) Dynamique écologique et typologie des territoires pastoraux des Alpes du Nord. 2. Analyse des facteurs de la valeur pastorale. Acta Oecol Oecol App/ 8, 283-300

Dorioz JM, Party JP (1987) Dynamique écologique et typologie des territoires pastoraux des Alpes du Nord. 1. Analyse de l'organisation agro-écologique d'un secteur de référence. Acta Oecol Oecol Appl 6, 257-280

Dubost M, Bornard A (1986) Étude des alpages laitiers. Réunion du CS du GIS «Alpes du Nord», sept 1986, CEMAGREF, Grenoble, $21 \mathrm{p}$

Fleury P, Dorioz JM, Jeannin B (1985) Influence $d u$ milieu physique et des pratiques agricoles sur la végétation des prairies de fauche des Hautes Vallées des Alpes du Nord. INRA, études et recherches $n^{\circ}$ $3,52 p$

Gardelle C, Guet J (1968) Enquête pastorale dans les Alpes françaises. CERAFER, INGRM. Grenoble $n^{\circ}$ $39,1100 \mathrm{p}$

Gensac $P$ (1979) Pelouse supraforestière du massif de la Vanoise. Contribution à l'étude écologique des groupements végétaux du Parc National de la Vanoise. Trav Sci Parc Nat Vanoise, X, $243 p$

Hillel D (1971) Soil and Water. Physical principles and processes. Acad Press, New York, $288 p$

Huguet E, Roybin D (1984) Diversité des systèmes de production en Beaufortain. Essai d'élaboration d'une typologie. Mémoire d'étude ESAA, Angers, $286 p$

Ismaïl SNA (1975) Micromorphometric soil porosity characterization by means of electro-optical image analysis (Quantimet 720). Soil Survey Pap 9, Wageningen, $104 \mathrm{p}$

Legros JP (1982) L'évolution granulométrique au cours de la pédogénèse. Approche par simulation sur ordinateur. Thèse Doct d'Etat, Univ Montpellier, $436 \mathrm{p}$

Legros JP, Robert M, Van Oort F (1980) Caractères principaux de la pédogénèse sur calcschiste en montagne. Sci Sol 4, 297-312

Legros JP, Party JP, Dorioz JM (1987) Répartition des milieux calcaires calciques et acidifiés en haute montagne calcaire humide. Conséquences agronomiques et écologiques. Doc Carto Écol, Grenoble, vol XXX, 137-157

Miedema R, Pape T, Van der Waal GJ (1974) A method to impregnate wet soil samples, producing high quality thin sections. Neth J Agric Sci 22, 3739

Monnier G, Stengel P, Fies JC (1973) Une méthode de mesure de la densité apparente de petits agglomérats terreux. Application à l'analyse des systèmes de porosité du sol. Ann Agron 24, 533-545

Naveh Z, Lieberman AS (1984) Landscape œcology. Theory and application. Springer Verlag, New York, $355 p$ 
Oort (van) F (1984) Géométrie de l'espace poral, comportement hydrique et pédogénèse. Application à des sols sous prairie et alpage, issus de l'altération des calcschistes sédimentaires en moyenne et haute montagne, Alpes du Nord (Beaufortain). Thèse Doc Ing INA-PG, $281 \mathrm{p}$

Oort (van) F, Tessier D, Robert M (1987) Méthodes d'observation et de mesure de la structure du sol. Application à la pédogénèse d'un sol développé sur calcshale. In: Micromorphologie des sols (N Fedoroff, LM Bresson, MA Courty, eds). Actes de la VIle Réunion Int Micromorphologie des Sols, Paris, $1985,521-528$

Oort (van) F, Robert M (1988) Rôle de l'oxydation du soufre dans les premiers stades de l'altération superficielle des calcschistes (Beaufortain, Alpes du Nord). Conséquences générales sur l'évolution pédologique. CR Séances Acad Sci Paris, t 307, série II, 819-825

Ozenda $\mathrm{P}$ (1985) La végétation de la chaine alpine dans l'espace montagnard européen. Masson, Paris, $344 p$
Richard L, Pautou G (1982) Carte de la végétation au 1/200 000. Alpes du Nord et Jura méridional. CNRS, Paris, $316 p$

Tessier D (1984) Étude expérimentale de l'organisation des matériaux argileux. Hydratation, gonflement et structuration au cours de la dessiccation et de la réhumectation. Thèse, Paris $\mathrm{VI}, 361 \mathrm{p}$

Tessier D, Berrier J (1979) Utilisation de la microscopie électronique à balayage dans l'étude des sols. Observation de sols humides soumis à différents pF. Sci Sol 1, 67-82

Tricart J (1978) Géomorphologie applicable. Masson, Paris, $204 p$

Vachier P, Cambier P, Prost R (1979) Structure d'un milieu poreux : la craie. Ann Agron 39, 247-263

Vertes $F$ (1983) Contribution à l'étude phytosociologique et écologique des prairies et alpages de moyenne Tarentaise. Application à l'évaluation des potentialités fourragères de la vallée de Pesey Nancroix. Thèse INAPG, Paris, $160 p$

Veyret P, Veyret G (1979) Atlas et géographie des Alpes françaises. Flammarion, $315 \mathrm{p}$ 